\title{
Metallic Phase in Quantum Hall Systems due to Inter-Landau-Band Mixing
}

\author{
Gang Xiong, ${ }^{1,2}$ Shi-Dong Wang, ${ }^{1}$ Qian Niu, ${ }^{3}$ De-Cheng Tian, ${ }^{2}$ and X. R. Wang ${ }^{1}$ \\ ${ }^{1}$ Physics Department, The Hong Kong University of Science and Technology, Clear Water Bay, Hong Kong SAR, China \\ ${ }^{2}$ Physics Department, Wuhan University, Wuhan, Hubei 430072, China \\ ${ }^{3}$ Physics Department, The University of Texas at Austin, Austin, Texas 78712-1081
}

(Received 22 February 2001; published 1 November 2001)

\begin{abstract}
The electronic eigenstates of a quantum Hall $(\mathrm{QH})$ system are chiral states. Strong inter-Landau-band mixings among these states can occur when the bandwidth is comparable to the spacing of two adjacent Landau bands. We show that mixing of localized states with opposite chirality can delocalize electronic states. Based on numerical results, we propose the existence of a metallic phase between two adjacent $\mathrm{QH}$ phases and between a $\mathrm{QH}$ phase and the insulating phase. This result is consistent with nonscaling behaviors observed in recent experiments on a quantum Hall liquid-to-insulator transition.
\end{abstract}

DOI: $10.1103 /$ PhysRevLett.87.216802

Recently there has been a great renewal of interest on transitions from integer quantum Hall effect (IQHE) states to an insulator [1-5]. According to the scaling theory of localization [6], all electrons in a disordered two-dimensional system are localized in the absence of a magnetic field. In the presence of a strong magnetic field, a series of disorder-broadened Landau bands (LBs) will appear, and extended states reside at the centers of these bands while states at other energies are localized. The integrally quantized Hall $(\mathrm{QH})$ plateaus are observed when the Fermi level lies in the localized states, with the value of the Hall conductance, $\sigma_{x y}=n e^{2} / h$, related to the number of filled LBs $(n)$. As a function of the magnetic field, the Hall conductance jumps from one QH plateau to another when the Fermi energy crosses an extended-state level. Many previous studies [1-5] have been focused on how such a transition occurs.

One overlooked issue regarding IQHE is the nature of a transition from one $\mathrm{QH}$ plateau to another. All existing theories assume it to be a continuous quantum phase transition. The fingerprint of a continuous phase transition is scaling laws around the transition point, and this assumption is mainly due to the early scaling experiments [7]. In the case of IQHE, a continuous quantum phase transition means algebraic divergence of the longitudinal Hallresistivity slope in temperature $T$ at the transition point. However, recent experiments [5] showed that such slopes remain finite when they are extrapolated to $T=0$. This implies a nonscaling behavior around a transition point, contradicting the expectation of continuous quantum phase transitions suggested by the theories. It also means that one should reexamine the nature of plateau transitions.

In this Letter we show that a narrow metallic phase may exist between two adjacent IQHE phases and between an IQHE phase and an insulating phase when the effect of interband mixing of opposite chirality is taken into account. Thus, it corresponds to two consecutive quantum phase transitions instead of one when the Hall conductance jumps from one plateau to another, consistent with the nonscaling behavior observed in experiments.
PACS numbers: 73.43.-f, 71.30.+h, 73.20.Jc

According to the semiclassical theory [8], an electronic state in a strong magnetic field and in a smooth potential can be decomposed into a rapid cyclotron motion and a slow drifting motion of the guiding center. The kinetic energy of the cyclotron motion is quantized by $E_{n}=(n+$ $1 / 2) \hbar \omega_{c}$, where $\omega_{c}$ is the cyclotron frequency and $n$ is the Landau band index. The trajectory of the drifting motion of a guiding center is along an equipotential contour of value $V_{0}=E-E_{n}$, where $E$ is the total energy of the electron. The equipotential contour consists of many loops, and each loop corresponds to one quantum state. The loops are localized around potential valleys for $V_{0}<0$ and around peaks for $V_{0}>0$. The drifting direction of each loop is unidirectional, i.e., chiral states. If one views the plane from the direction opposite to the magnetic field, the drifting is clockwise around valleys and counterclockwise around peaks. In the absence of inter-Landau-band mixing, it has been shown that [8] extended states reside only at $V_{0}=0$, i.e., at the center of each LB.

In a weak magnetic field or strong disorders, the width of the Landau subbands may be comparable with the Landau gap, and interband mixing can no longer be ignored. In order to investigate the consequences of this mixing, we consider a simple system of two adjacent LBs. Our interest will be on the localization properties of electronic states between the two bands. Using the semiclassical theory described in the previous paragraph, two sets of loops are obtained, one for the upper LB and the other for the lower LB. The two sets have opposite chirality, and they are spatially separated. The set of loops in the upper band is localized around valleys denoted by V in Fig. 1(a) and the other set around peaks denoted by P. Assuming that tunneling can occur between two neighboring localized states (loops) from the same band near their saddle point and two nearby states of different bands are coupled due to impurity scattering, Fig. 1(a) is topologically equivalent to a two-channel version of the Chalker-Coddington (CC) network model [9], as shown in Fig. 1(b). In a one-channel CC network model, one, and only one, extended state exists in every LB center, corresponding to the percolation point. 


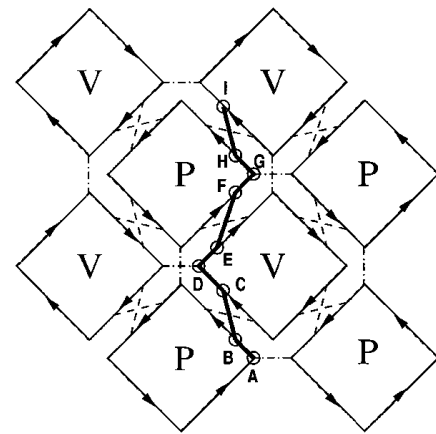

(a)

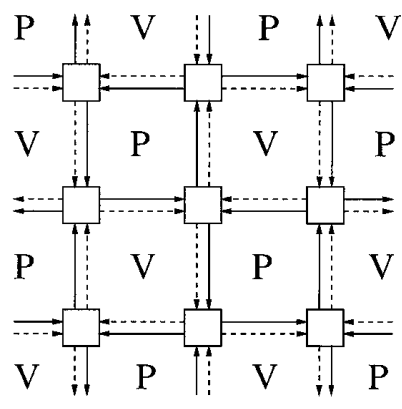

(b)
FIG. 1. (a) Topological plot of the trajectories of the drifting motion of guiding centers (rhombus). The drifting motions around a potential peak (valley) are denoted by $\mathrm{P}(\mathrm{V})$, and their directions are indicated by the arrows. The dashed lines stand for interband mixing, and the dotted lines stand for tunneling at saddle points. The thick line ( $A$ to $I)$ describes the trajectory of an electron due to a strong interband mixing. (b) The equivalent two-channel Chalker-Coddington network model of (a). The solid and dashed lines on each link denote two channels from two LBs. The squares stand for saddle points. P, V, and arrows have the same meaning as those in (a).

If we turn on the interband mixing of the opposite chirality, localized loops may become less localized. In order to see this, let us consider an extreme case with no tunneling at saddle points, but with such a strong interband mixing that an electron will move from a loop, say, around a peak, to a neighboring loop around a valley [as shown by $B \rightarrow C$ in Fig. 1(a)]. Following an electron starting at $A$, the trajectory of this electron will be $A \rightarrow B \rightarrow C \rightarrow D \rightarrow E \ldots$. The electron no longer moves on a delocalized closed loop. On the other hand, for two sets of loops with the same chirality (that is, both of them are localized around either valleys or peaks), the interband mixing mainly occurs between two loops localized around the same position, and this mixing cannot help much to delocalize a state. This is why we consider the mixing between spatially separated states with opposite chirality, but neglect the mixing between states of the same chirality separated only in energy. However, it does not mean that the mixing of the same chirality has no effect at all. As was found in some previous works [9], this type of mixing may shift an extended state from its LB center. Level shifting due to mixing between states of the same chirality may distort the shape of the phase diagram, but should not alter its topology. The merging of the bands of extended states is exclusively due to the mixing between states of opposite chirality.

The electron localization length is often obtained from the transfer matrix method. For a two-dimensional system, however, it is well known that, from this quantity, it is difficult to provide a conclusive answer to questions related to the metal-insulator transition (MIT) [10]. On the other hand, level-statistics analysis [11] has been used in studying MIT. We follow the approach proposed by Klesse and Metzler [11]. A quantum state of the network model can be expressed by a vector $\vec{\Phi}=\left(\left\{\phi_{i}^{u}, \phi_{i}^{l}\right\}\right)$, where $\phi_{i}^{u}$ and $\phi_{i}^{l}$ are the wave function amplitudes of the $i$ th link of the upper band $(u)$ and the lower band $(l)$, respectively. As shown by Fertig [12], the network model can be described by an evolution operator $U(E)$ which is an $E$-dependent matrix determined by the scattering properties of nodes and links in the model. The eigenvalue equation of this operator is $U(E) \vec{\Phi}_{\alpha}(E)=e^{i \omega_{\alpha}(E)} \vec{\Phi}_{\alpha}(E)$, where $\alpha$ is the eigenstate index. The eigenenergies $\left\{E_{n}\right\}$ of the system are those $E$ 's with $\omega$ being integer multiples of $2 \pi$. It was shown [11] that the level-spacing statistics of the set of quasienergies $\left\{\omega_{\alpha}(E)\right\}$ is the same as that of the set of $\left\{E_{n}\right\}$ near $E$. Therefore, the localization property of states with an energy $E$ can be obtained. The great advantage of this approach is that all the eigenvalues of the evolution operator $U(E)$ can be used in the analysis. A characteristic quantity $I_{0}$, defined by $I_{0}=\int s^{2} P(s) d s / 2$, is used to examine the localization property, where $P(s)$ is the level-spacing distribution function of the quasienergies [11]. It is well known that $I_{0}=1$ for localized states [11]. Thus, we use the following simple criteria. If $I_{0}$ of a state with energy $E$ increases and approaches 1 with lattice size $L$, this state is localized. Otherwise, it is extended. We studied the model for $L=8,12,16$ (and 20 for some energy values) with periodic boundaries along both directions. For each energy value $E$, a sufficient number of ensembles are used in order to collect more than $5 \times 10^{4}$ data points in our analysis.

In the following analysis, we assume that interband mixing takes place only on the links. Tunneling at each node occurs for the same band and is described by a $\mathrm{SO}(4)$ matrix,

$$
S=\left(\begin{array}{cccc}
s_{u}^{R} & s_{u}^{L} & 0 & 0 \\
-s_{u}^{L} & s_{u}^{R} & 0 & 0 \\
0 & 0 & s_{l}^{R} & s_{l}^{L} \\
0 & 0 & -s_{l}^{L} & s_{l}^{R}
\end{array}\right)
$$

where the subscript $u$ represents the upper band and $l$ represents the lower LBs. The elements $s_{u(l)}^{L}$ and $s_{u(l)}^{R}$ are related to the scattering probabilities of an incoming wave function in the upper (lower) band of the outgoing channels on its left- and right-hand sides, respectively. We choose $s_{u(l)}^{R}=\sqrt{1-\left(s_{u(l)}^{L}\right)^{2}}$ because of the orthogonality of the matrix. The potential around a saddle point is assumed to be $V(x, y)=-U x^{2}+U y^{2}+V_{c}$ [9], where $U$ is a constant describing the potential fluctuation and $V_{c}$ is the potential barrier at this point. The left-hand scattering amplitude is given by [12]

$$
s_{u(l)}^{L}=\left[1+\exp \left(-\pi \epsilon_{u(l)}\right)\right]^{-1 / 2},
$$

where $\epsilon_{u(l)}=\left[E+V_{c}-\left(n_{u(l)}+1 / 2\right) E_{2}\right] / E_{1}, E$ is the electronic energy, $E_{1}=\frac{\hbar \omega_{c}}{2 \sqrt{2}} \sqrt{K-1}$, and $E_{2}=\frac{\hbar \omega_{c}}{\sqrt{2}} \times$ $\sqrt{K+1}$, with $K=\sqrt{\frac{64 U^{2}}{m^{2} \omega_{c}^{4}}+1}$. The kinetic energies of 
cyclotron motion in the two bands are $\left(n_{u}+1 / 2\right) E_{2}$ and $\left(n_{l}+1 / 2\right) E_{2}$, respectively, where $n_{u(l)}$ are the band indices and $\Delta n=n_{u}-n_{l}=1$. The dimensionless ratio $E_{r}=E_{2} / E_{1}=2 \sqrt{1+\frac{2}{K-1}}$ approaches 2 from above as $U$ or the inverse of $\omega_{c}$ increases [12], i.e., the strong disorder or weak-field regime. Since this is the regime we are interested in, we choose the value of it to be 2.2 in our calculations. For convenience, we choose $E_{2}$ as the energy unit and the cyclotron energy of the lower band as the reference point. The energy range between the two band centers is thus $E \in[0,1]$. The potential $V_{c}$ at a saddle point is treated as a random number uniformly distributed within the range of $\left[-E_{1}, E_{1}\right]$.

Interband mixing on a link is described by a $\mathrm{U}(2)$ matrix with random Aharonov-Bohm phases $\phi_{i}(i=1,2,3,4)$ accumulated along propagation paths,

$$
M=\left(\begin{array}{cc}
e^{i \phi_{1}} & 0 \\
0 & e^{i \phi_{2}}
\end{array}\right)\left(\begin{array}{cc}
\cos \theta & \sin \theta \\
-\sin \theta & \cos \theta
\end{array}\right)\left(\begin{array}{cc}
e^{i \phi_{3}} & 0 \\
0 & e^{i \phi_{4}}
\end{array}\right)
$$

where $\sin \theta$ describes the mixing strength. In our calculations, we assume that they are uniformly distributed in $[0,2 \pi][8]$. $P$, defined as $\sqrt{P /(1+P)}=\sin \theta$, is used to characterize the mixing strength. $P$ will take the same value for all links in our calculations.

The curves in Fig. 2 are $I_{0}$ vs mixing strength $P$ at $E=$ 0.02 (a), $E=0$ [inset of (a)], and $E=0.5$ (b) for $L=8$, $12,16,20$. The state of $E=0.02$ is localized at zero mixing since $I_{0}$ increases with sample size $L$. Figure 2(a) shows two crossing points at small $P$. Between the two points, $I_{0}$ decreases with sample size $L$. Thus, according to the criteria explained earlier, the state is extended in this regime. For the lower band center $E=0, I_{0}$ decreases initially with $L$ at small $P$, indicating an extended state. Then $I_{0}$ of different $L$ crosses at a particular $P_{c}$. $I_{0}$ decreases with $L$ for $P>P_{c}$, showing the feature of a localized state. Figure 2(b) shows that the state of $E=0.5$ is localized at small $P$ because $I_{0}$ increases with sample size and approaches 1 . For large $P(>1)$, all curves of a differ-

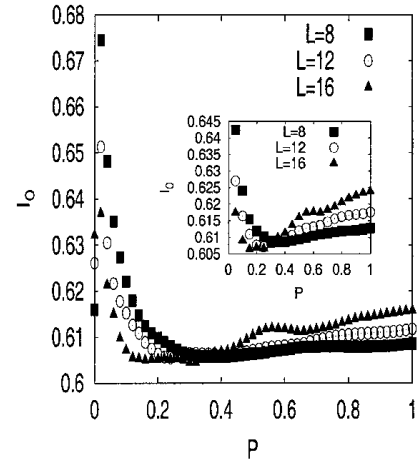

(a)

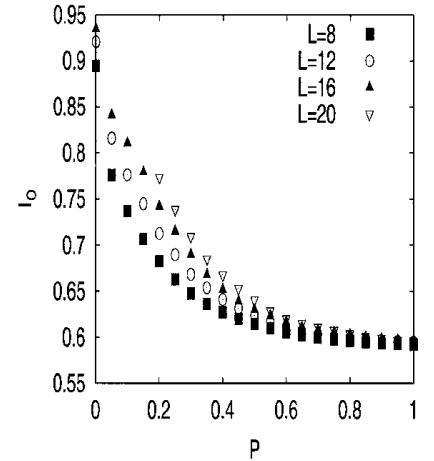

(b)
FIG. 2. $I_{0}$ vs $P$ for $L=8,12,16$ : (a) $E=0.02$ (inset, $E=0$ ); (b) $E=0.5$. ent system size tend to merge together. The independence of $I_{0}$ on system size suggests a localization-delocalization transition at the merging point.

The existence of new extended states at $E \sim 0.5$ in the case of strong interband mixing can be understood as follows. Assuming that the intraband tunneling at nodes are negligibly weak for states of $E \sim 0.5$, we see from Fig. 1(a) that the maximal interband mixing $(\sin \theta=1)$ delocalizes the state, which is localized at zero interband mixing. If one views $p=\sin ^{2} \theta$ as a connection probability of two neighboring loops of opposite chirality, our twochannel model without intraband tunnelings at nodes is analogous to a bond-percolation problem. It is well known that a percolation cluster exists at $p \geq p_{c}=1 / 2$ or $P \geq$ $P_{c}=1$ for a square lattice [13]. Therefore, an extended state is formed by strong mixing. One hopes that the intraband tunnelings at nodes will modify only the threshold value of the mixing strength.

To express our numerical results in the $E-P$ plane, a topological phase diagram shown in Fig. 3(a) is obtained. In the absence of interband mixing, only the singular energy level of each LB is extended. In the presence of interband mixing of opposite chirality, there are two regimes. At weak mixing when the inter-Landau-band separation is much smaller than the Landau bandwidth, each of the extended states broadens to a narrow band of extended states near the LB centers. The extended states in the lowest LB shift from the LB center. These extended states will eventually merge with those from the higher LBs. This shifting of extended states was also observed previously [1]. At strong mixing, a band of extended states exists between two Landau bands, where all states are localized without the mixing. Systems in a weak magnetic field should correspond to the strong mixing case. In terms of the $\mathrm{QH}$ plateau transitions, a direct transition occurs because two $\mathrm{QH}$ phases merge together to form a narrow metallic phase in a weak field. Thus, we propose that a direct transition

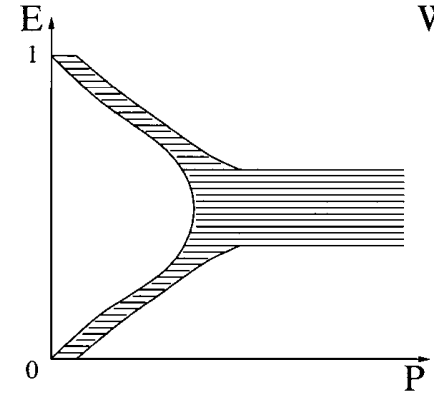

(a)

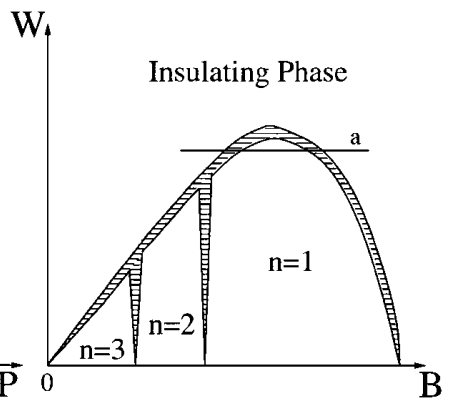

(b)
FIG. 3. (a) Topological phase diagram of electron localization in the $E-P$ plane. The shadowed region is the extended states (metallic phase). (b) Topological $\mathrm{QH}$ phase diagram in the $W-B$ plane. $W$ stands for the disorder strength, and $B$ stands for the magnetic field. The shadowed region is the metallic phase. The area indicated by the symbol $n$ is the $n$-plateau IQHE phase. The rest of the area is the insulating phase. 
from an IQHE phase to the insulating phase is realized by passing through a metallic phase.

Plot above results in the plane of disorder and magnetic field; we obtain a new topological $\mathrm{QH}$ phase diagram [as shown in Fig. 3(b)]. This is similar to the empirical diagram obtained experimentally in Ref. [4]. The origin $(W=0, B=0)$ is a singular point. According to the weak localization theory [6], no extended state exists at this point. Differing from existing theories, there exists a narrow metallic phase between two adjacent IQHE phases and between an IQHE phase and an insulating phase. This new phase diagram is consistent with the nonscaling experiments [5], where samples are relatively dirty and interband mixing is strong, corresponding to a process along line $a$ in Fig. 3(b). The system undergoes two quantum phase transitions each time it moves from the $\mathrm{QH}$ insulating phase to the IQHE phase of $n=1$ and back to the Anderson insulating phase as the magnetic field decreases. To verify this claim, we analyzed the original experimental data in Ref. [6] according to the two quantum phase transition point assumptions. The standard one-parameter scaling analysis is performed, and good scaling behaviors are obtained for two close critical filling factors of $\nu_{c 1}=$ 0.6453 and $\nu_{c 2}=0.6477$. The critical exponents in the left- and right-hand sides of the transition regime are equal and the value is $z \nu=2.33 \pm 0.01$. This value is consistent with previous works [7-9]. On the other hand, if we are forced to fit the data by one critical point in each transition, we find either two distinct values for critical exponents on the two sides of the critical point or the data do not follow the scaling law at all. Our fitting shows that the width of the metallic phase regime is about $5 \times 10^{-3} \mathrm{~T}$ while the value of the magnetic field was increased by $1 \sim 2 \times 10^{-3} \mathrm{~T}$ each time in the experiments. This may explain why the metallic phase was overlooked.

It is worth noting that two metallic states have been studied extensively in the $\mathrm{QH}$ system. One is the composite fermion state at the half filling in the lowest Landau level (LL) and the other is the stripe state at the half-filled higher LLs. These states are formed by the Coulomb interaction effect in the high mobility samples. They are different from our metallic phase due to level mixing. Although we have not considered the electron-electron interactions in our study, there is no reason why the delocalization effect due to the level mixing would be destroyed by the Coulomb interaction. Of course, the interaction could cause the level mixing effect with a different dependence on the magnetic field. Our model can be used to describe spin polarized systems. In this case, the two LBs are for spin up and spin down states, respectively. Indeed, two-channel CC models have been used previously to simulate spin resolved problems [9]. These studies cannot distinguish an extended state from a localized state between the energy region of the two extended states of the two Landau bands. In this sense, our results are consistent with those of early works.
In summary, we state that inter-Landau-band mixing of opposite chirality can lead to delocalization. Within the network model, we found numerical evidence for a metallic phase between two neighboring $\mathrm{QH}$ phases by using the level-spacing statistics analysis. This new phase diagram gives a possible explanation for nonscaling behavior observed in recent experiments. The results of a scaling analysis on experimental data support our conclusion. We also point out the difference between our results and those of Wang et al. We have considered only two LBs in this work, but we believe that metallic phases exist in higher IQHE phases. But, further study including more LBs is needed to answer this question definitively.

This work was supported by the Research Grant Council of HKSAR, China. Q. N. acknowledges the support of NSF-DMR9705406, the Welch Foundation, and China-NSF. G.X. thanks Dr. Dan Shahar for providing the original experimental data.

[1] S. Kivelson, D. H. Lee, and S. C. Zhang, Phys. Rev. B 46, 2223 (1992); D. Z. Liu, X. C. Xie, and Q. Niu, Phys. Rev. Lett. 76, 975 (1996); D. N. Sheng and Z. Y. Wang, Phys. Rev. Lett. 80, 580 (1998); X. R. Wang, X. C. Xie, Q. Niu, and J. Jain, cond-mat/0008411.

[2] H. W. Jiang, C. E. Johnson, K. L. Wang, and S. T. Hannahs, Phys. Rev. Lett. 71, 1439 (1993); I. Glozman, C. E. Johnson, and H. W. Jiang, Phys. Rev. Lett. 74, 594 (1995).

[3] D. Shahar, D. C. Tsui, and J. E. Cunningham, Phys. Rev. B 52, R14 372 (1995); S.-H. Song, D. Shahar, D. C. Tsui, Y.H. Xie, and Don Monroe, Phys. Rev. Lett. 78, 2200 (1997).

[4] S. V. Kravchenko, W. E. Mason, J. E. Fureaux, and V. M. Pudalov, Phys. Rev. Lett. 75, 910 (1995).

[5] M. Hilke et al., Phys. Rev. B 56, 15545 (1997); D. Shahar et al., Solid State Commun. 107, 19 (1998).

[6] E. Abrahams et al., Phys. Rev. Lett. 42, 673 (1979).

[7] H. P. Wei et al., Phys. Rev. Lett. 61, 1294 (1988).

[8] J. T. Chalker and P. D. Coddington, J. Phys. C 21, 2665 (1988); A. G. Galstyan and M. E. Raikh, Phys. Rev. B 56, 1422 (1997).

[9] Z. Q. Wang, D. H. Lee, and X. G. Wen, Phys. Rev. Lett. 72, 2454 (1994); D. K. K. Lee and J. T. Chalker, Phys. Rev. Lett. 72, 1510 (1994); V. Kagalovsky, B. Horovitz, and Y. Avishai, Phys. Rev. B 52, 17044 (1995).

[10] X. C. Xie, X. R. Wang, and D. Z. Liu, Phys. Rev. Lett. 80, 3563 (1998).

[11] R. Klesse and M. Metzler, Phys. Rev. Lett. 79, 721 (1997); M. Metzler and I. Varga, J. Phys. Soc. Jpn. 67, 1856 (1998); M. L. Mehta, Random Matrices (Academic, New York, 1991), 2nd ed.

[12] H. A. Fertig and B. I. Halperin, Phys. Rev. B 36, 7969 (1987); H. A. Fertig, Phys. Rev. B 38, 996 (1988).

[13] D. Stauff and A. Aharony, Introduction to Percolation Theory (Taylor \& Francis, London, 1994). 